\title{
ALFREDO BERMUDEZ \\ CARMEN RODRIGUEZ \\ A nonlinear evolution equation modelling the Marangoni effect : existence of solution and numerical methods
}

Annales de la faculté des sciences de Toulouse $5^{e}$ série, tome $8, \mathrm{n}^{\circ} 2$ (1986-1987), p. 205-223

<http://www.numdam.org/item?id=AFST_1986-1987_5_8_2_205_0>

(C) Université Paul Sabatier, 1986-1987, tous droits réservés.

L'accès aux archives de la revue «Annales de la faculté des sciences de Toulouse » (http://picard.ups-tlse.fr/ annales/) implique l'accord avec les conditions générales d'utilisation (http://www.numdam.org/conditions). Toute utilisation commerciale ou impression systématique est constitutive d'une infraction pénale. Toute copie ou impression de ce fichier doit contenir la présente mention de copyright.

\section{NumDam}

Article numérisé dans le cadre du programme Numérisation de documents anciens mathématiques http://www.numdam.org/ 


\title{
A nonlinear evolution equation modelling the Marangoni effect : existence of solution and numerical methods
}

\author{
ALFREDO BERMUdEZ ${ }^{(1)}$ AND CARMEN RODRIGUEZ ${ }^{(1)}$
}

Resumb. - Dans cet article on démontre un théorème d'existence et unicité pour une équation d'évolution abstraite, avec un opérateur nonlinéaire dépendant du temps. Le résultat s'applique à une équation aux dérivées partielles modélisant l'effet Marangoni pour un fluide non-newtonien. On présente aussi une méthode de résolution numérique qui est appliquée à des exemples tests.

ABstract. - Existence and uniqueness of solution of an evolution equation with a nonlinear operator depending on time is proved. The result is applied to a boundary value problem modelling the Marangoni effect in a non-newtonian fluid. Numerical solution is also considered.

\section{Introduction}

In this paper we prove an existence and uniqueness theorem for a nonlinear evolution equation in a Hilbert space of the type

$$
\frac{d u}{d t}(t)+A(u(t))+F(t) B \partial \varphi\left(B^{*} u(t)\right) \ni f(t)
$$

where $A$ is a nonlinear monotone operator, $\varphi$ is a lower semi-continuous convex function, $B$ is a bounded linear operator and $F$ is a function.

This type of equations appears when considering a mathematical model of the Marangoni effect in a non newtonian viscous fluid.

(1) Departamento de Matematica Aplicada, Facultad de Matematicas, Universidad de Santiago de Compostela, Santiago de Compostela - Espagne 


\section{A. Bermudez and C. Rodriguez}

Evolution equations with nonlinear operators depending on time have been studied in many articles : Kato [9], Crandall and Pazy [8], Watanabe [14], Peralba [12], Kenmochi [10]; Attouch and Damlamian [1], [2], Bermudez, Durany and Saguez [6].

However, the results given in these papers cannot be applied to our situation. In particular, the assumptions required in Atrouch and Damlamian [1] are not satisfied in the case of the Marangoni effect.

In the present paper we first recall a mathematical model for the Marangoni effect in a non-newtonian viscous fluid and obtain a variational formulation which corresponds to a particular case of (1.1).

Then we prove an existence and uniqueness theorem. For this particular case we approximate the problem and use existence results from ATTOUCH and Damlamian [1]. Some a priori estimates allow passing to the limit.

\section{The physical problem}

If a small drop of a soluble or partially soluble liquid having a smaller surface tension than that of water is put on a free water-air interface, a velocity field develops because of the surface tension gradient. This is the most simple manifestation of the Marangoni effect.

From the mass and momentum conservation equations, assuming cylindrical symmetry and after some simplifications, RUCKENSTEIN, SMIGELSCHI and SUCIU [13] obtained the following mathematical model for the radial velocity $u$ :

$$
\begin{cases}\frac{\partial u}{\partial t}(t, x)-\frac{\partial}{\partial x}\left(\nu\left|\frac{\partial u}{\partial x}(t, x)\right|^{p-2} \frac{\partial u}{\partial x}(t, x)\right)=0 & 0<t<T \\ \nu\left|\frac{\partial u}{\partial x}(t, x)\right|^{p-2} \frac{\partial u}{\partial x}(t, x)=-\frac{F(t)}{u(t, x)} & 0<t<T \\ u(t, L)=0 & x=0 \\ u(0, x)=0 & 0<t<T \\ & 0<x<L\end{cases}
$$

where $\nu$ and $p$ are given constants, and $F$ is a given function all depending on the fluid (the case $p=2$ corresponds to a newtonian fluid).

Note that if $u$ is a solution of $(2.1),-u$ is also a solution. Hence, we do not have uniqueness.

On the other hand and from the physical point of view we are interested in positive solutions, and any positive solution of $(2.1)$ is a solution of the problem : 
Find a function $u$ such that

$$
\begin{cases}\frac{\partial u}{\partial t}(t, x)-\frac{\partial}{\partial x}\left(\nu\left|\frac{\partial u}{\partial x}(t, x)\right|^{p-2} \frac{\partial u}{\partial x}(t, x)\right)=0 & 0<t<T \\ \nu\left|\frac{\partial u}{\partial x}(t, x)\right|^{p-2} \frac{\partial u}{\partial x}(t, x) \in F(t) G(u(t, x)) & 0<x<L \\ u(t, L)=0 & x=0 \\ u(0, x)=0 & 0<t<T \\ & 0<x<L\end{cases}
$$

Where $G$ is the maximal monotone operator (see for instance BREZIs [7]) in $\mathbf{R}$ given by

$$
G(x)= \begin{cases}0 & x \leq 0 \\ -\frac{1}{x} & x>0\end{cases}
$$

Conversely, any solution of (2.2) is a positive solution of (2.1).

Multiplying by test functions and integrating by parts, the following variational formulation can be obtained.

Find $u$ such that

$$
\left\{\begin{aligned}
&\left(\frac{d u}{d t}(t),\right.z-u(t))+\phi(z)-\phi(u(t))+F(t) \varphi(z(0))-F(t) \varphi(u(t, 0)) \geq 0 \\
& \forall z \in W^{1, p}(0, L) \text { with } z(L)=0 \text { and a.e. on }(0, T) \\
& u(t, L)=0 \\
& u(0, x)=0
\end{aligned}\right.
$$

where

$$
\varphi(x)=\left\{\begin{array}{cc}
\infty & x \leq 0 \\
-\ln x & x>0
\end{array} \quad x \in \mathbf{R}\right.
$$

and

$$
\phi(v)=\frac{\nu}{p} \int_{0}^{L}\left|\frac{\partial v}{\partial x}\right|^{p} d x, v \in W^{1, p}(0, L)
$$

The variational inequality (2.4) is a week formulation of problem (2.2).

\section{A nonlinear differential equations : existence of solution}

Let $V$ be a reflexive separable Banach space. Let $E$ and $H$ be Hilbert spaces. We shall assume that $V$ is dense in $H$ and that the inclusion of $V$ 


\section{A. Bermudez and C. Rodriguez}

in $H$ is compact. If we identify $H$ with its topological dual, we have the following classical inclusions :

$$
V \subset H \subset V^{\prime}
$$

Denote by \|\| and \|\|$_{*}$ the norms of $V$ and $V^{\prime}$, respectively, and by || the norm in $H$.

In addition, we shall suppose :

(H1) $B \in L\left(E, V^{\prime}\right)$

(H2) $\varphi: E \rightarrow(-\infty,+\infty]$ is a lower-semicontinuous (1.s.c.) proper and convex function.

(H3) $\phi: V \rightarrow \mathbf{R}$ is a differentiable functional in the Gateaux sense.

We consider in $V$ a seminorm [] that satisfies

(H4) There exists $\lambda>0$ and $\beta>0$ such that

$$
[v]+\lambda|v| \geq \beta\|v\| \quad \forall v \in V
$$

(H5) There exists $\alpha>0$ such that

$$
\phi(v) \geq \alpha[v]^{p} \quad \forall v \in V, \quad 1<p<\infty
$$

(H6) $f \in L^{2}(0, T ; H)$ and $u_{o} \in H$

(H7) $\quad \operatorname{Im} \Lambda_{E}^{-1} B^{*} \cap \operatorname{dom}(\varphi) \neq \emptyset$

(H8) $F \in C^{1}[0, T]$ with $F(t)>0 \forall t>0$ and $F(0)=0$.

We now consider the following problem : find a function $u$ such that

$$
\left\{\begin{array}{l}
\left(\frac{d u}{d t}(t), z-u(t)\right)+\phi(z)-\phi(u(t))+F(t) \varphi\left(\Lambda_{E}^{-1} B^{*} z\right)- \\
\quad-F(t) \varphi\left(\Lambda_{E}^{-1} B^{*} u(t)\right) \geq(f(t), z-u(t)), \forall z \in V \text { and a.e. on }(0, T) \\
u(0)=u_{0}
\end{array}\right.
$$

where $B^{*}$ is the adjoint operator of $B$ and $\Lambda_{E}$ the canonical isomorphism from $E$ into $E^{\prime}$.

Remark 1. - If we take $V=\left\{z \in W^{1, p}(\Omega), z(L)=0\right\}$ with $\Omega=$ $(0, L), H=L^{2}(\Omega), E=\mathbf{R}, B$ given by :

$$
B(a)(v)=a v(0), \quad a \in E, \quad v \in V,
$$


$f=0, u_{0}=0$, and $\varphi$ and $\phi$ defined as in (2.5) and (2.6) respectively, then the problem (3.1) becomes the variational inequality (2.4).

We shall prove the following result.

THEOREM 1. - Under the hypothesis (Hi) - (H8), there exists a unique element $u$ solution of (9.1) such that

$$
\begin{aligned}
& u \in L^{p}(0, T ; V) \cap L^{\infty}(0, T ; H) \\
& \sqrt{F} \frac{d u}{d t} \in L^{2}(0, T ; H)
\end{aligned}
$$

Moreover if $u_{0} \in \operatorname{dom}(\varphi)$, then $d u / d t \in L^{2}(0, T ; H)$.

To prove this theorem, we will use the following result from ATTOUCH and Damlamian [1] :

THEOREM 2. - Let $H$ be a real Hilbert space and $(\psi(t, .))_{t \in[0, T]}$ a family of l.s.c. proper convex functions from $H$ in $(-\infty,+\infty]$ satisfying :

(I) $\operatorname{dom}(\psi(t,))=$.$D is independent of t$.

(II) $\forall r>0 \exists C_{r} \geq 0$ and $a_{r} \in W^{1,1}(0, T ; R)$ such that

$$
\begin{aligned}
\forall x \in & D,|x| \leq r \text { and } \forall s, t \in[0, T]|\psi(t, s)-\psi(s, x)| \leq \\
& \leq\left|a_{r}(t)-a_{r}(s)\right|\left(g(t, x)+C_{r}\right) .
\end{aligned}
$$

Then, given $u_{0} \in \bar{D}$ and $f \in L^{2}(0, T ; H)$, there exists a unique classical solution of

$$
\frac{d u}{d t}+\partial \psi(t, u(t)) \ni f(t), u(0)=u_{0}
$$

Moreover

$$
\sqrt{t} \frac{d u}{d t} \in L^{2}(0, T ; H) \text { and } \forall t>0, u(t) \in D
$$

If $u_{0} \in D$ then

$$
\frac{d u}{d t} \in L^{2}(0, T ; H)
$$

and the map $t \rightarrow \psi(t, u(t))$ is absolutely continuous on $[0, T]$.

Remark 2. - In (3.2), $\partial \psi$ denotes the subdifferential of $\psi$ with respect to the second variable. Recall that

$$
v \in \partial \psi(t, u) \Longleftrightarrow \psi(t, \omega)-\psi(t, u) \geq(v, \omega-u)
$$

(see $[3],[7])$. 
Remark 3. - Notice that the assumption (II) is not satisfied by the functional

$$
\psi(t, u)=\phi(u)+F(t) \varphi\left(\Lambda_{E}^{-1} B^{*}(u)\right)
$$

because $F(0)=0$. Therefore, theorem 2 cannot be directly applied to the variational inequality (3.1).

Proof of Theorem 1. - Existence : Let $D$ be defined by

$$
D=\left\{u \in V: \Lambda_{E}^{-1} B^{*} u \in \operatorname{dom}(\varphi)\right\}
$$

If $u_{0} \in \bar{D}$ then there exists a sequence $\left\{u_{0_{n}}\right\}$ of elements in $D$ such that $\lim _{n \rightarrow \infty} u_{0 n}=u_{0}$.

We define the function $F^{n}$ by

$$
F^{n}(t)=F(t)+\frac{1}{\max \left\{n, \psi\left(0, u_{0 n}\right)\right\}}
$$

and the functional $\psi^{n}$ by

$$
\psi^{n}(t, u)=\phi(u)+F^{n}(t) \varphi\left(\Lambda_{E}^{-1} B^{*} u\right)
$$

From (H5) we have

$$
\psi^{n}(t, u(t))-\psi^{n}(s, u(s)) \leq(F(t)-F(s)) \frac{1}{\min F^{n}} \psi^{n}(t, u(t))
$$

Thus, hypothesis (II) of theorem 2 holds and we can conclude that there exists a unique classical solution $u^{n}$ of

$$
\frac{d u}{d t}+\partial \psi^{n}\left(t, u^{n}(t)\right) \ni f(t), u^{n}(0)=u_{O n} .
$$

Moreover

$$
\frac{d u^{n}}{d t} \in L^{2}(0, T ; H)
$$

and the map $t \rightarrow \psi^{n}\left(t, u^{n}(t)\right)$ is absolutely continuous on $[0, T]$.

A priori estimates I. - We first give the following.

LEMMA 1. - Let $v \in \operatorname{Im~} \Lambda_{E}^{-1} B^{*} \cap \operatorname{dom}(\varphi)$ (v exists by (H7)). Then, the function

$$
\begin{gathered}
\tilde{\varphi}(w)=\varphi(w)-\varphi(v)-(y, w-v) \\
-210-
\end{gathered}
$$


where $y \in \partial \varphi(v)$, is l.s.c. proper and convex.

Moreover $\tilde{\varphi} \geq 0, \tilde{\varphi}(v)=0$, and $\partial \tilde{\varphi}$ is given by

$$
\partial \tilde{\varphi}(w)=\partial \varphi(w)-y
$$

Since $u^{n}$ is a solution of $(3.10)$, we have

$$
\left\{\begin{array}{l}
\left(\frac{d u^{n}}{d t}(t), u^{n}(t)-z\right)+\phi\left(u^{n}(t)\right)-\phi(z)+F^{n}(t) \varphi\left(\Lambda_{E}^{-1} B^{*} u^{n}(t)\right) \\
\quad-F^{n}(t) \varphi\left(\Lambda_{E}^{-1} B^{*} z\right) \leq\left(f(t), u^{n}(t)-z\right), \forall z \in V \\
u^{n}(0)=u_{0 n}
\end{array}\right.
$$

Let $\bar{z} \in V$ such that $\Lambda_{E}^{-1} B^{*}(\bar{z})=v$. For $z=\bar{z}$, add to both sides of the inequality (3.12) the term $-F^{n}(t)\left(y, u^{n}(t)-\bar{z}\right)$. Since $\left(-d \bar{z} / d t, u^{n}(t)-\bar{z}\right)=$ 0 , we can add it to the left hand side of (3.12).

Then we integrate betwen 0 and $s>0$. According to lemma 1 and taking into account (H1) and (H5), we obtain

$$
\begin{aligned}
& \frac{1}{2}\left|u^{n}(s)-\bar{z}\right|^{2}+\alpha \int_{0}^{s}\left[u^{n}(t)\right]^{p} d t+\int_{0}^{s} F^{n}(t) \tilde{\varphi}\left(\Lambda_{E}^{-1} B^{*} u^{n}(t)\right) d t \\
& \leq \frac{1}{2}\left|u_{0 n}-\bar{z}\right|^{2}+\int_{0}^{s} \phi(\bar{z}) d t+\int_{0}^{s}\left|f \| u^{n}(t)\right| d t+\int_{0}^{s}|f||\bar{z}| d t \\
& +\int_{0}^{s} F^{n}(t)\|y\|\left\|B^{*}\right\|\left\|u^{n}(t)\right\| d t+ \\
& +\int_{0}^{s} F^{n}(t)\|y\|\left\|B^{*}\right\|\|\bar{z}\| d t
\end{aligned}
$$

From (3.13) and H4 taking into account that $F^{n} \in C^{1}[0, T]$, and using Hölder's inequality, we can write :

$$
\begin{aligned}
& \left|u^{n}(s)\right|^{2}+c_{1} \alpha \int_{0}^{s}\left[u^{n}(t)\right]^{p} d t+c_{1} \int_{0}^{s} F^{n}(t) \tilde{\varphi}\left(\Lambda_{E}^{-1} B^{*} u^{n}(t)\right) d t \\
& \leq c_{2}+c_{3}\left(\int_{0}^{s}\left[u^{n}(t)\right]^{p}\right)^{1 / p}+c_{4}\left(\int_{0}^{s}\left|u^{n}(t)\right|^{p}\right)^{1 / p}+ \\
& \quad+c_{5} \int_{0}^{s}\left|u^{n}(t)\right|^{2} d t
\end{aligned}
$$

where $c_{1}, c_{2}, c_{3}, c_{4}$ and $c_{5}$ are positive constants. 


\section{A. Bermudez and C. Rodriguez}

Now, by Young's inequality, we obtain :

$$
\begin{aligned}
& \left|u^{n}(s)\right|^{2}+c_{9} \int_{0}^{s}\left[u^{n}(t)\right]^{p}+c_{10} \int_{0}^{s} F^{n}(t) \tilde{\varphi}\left(\Lambda_{E}^{-1} B^{*} u^{n}(t)\right) d t \\
& \leq c_{6}+c_{7}\left(\int_{0}^{8}\left|u^{n}(t)\right|^{p} d t\right)^{1 / p}+c_{8} \int_{0}^{s}\left|u^{n}(t)\right|^{2} d t
\end{aligned}
$$

From this and using again Young's inequality we have

$$
\left|u^{n}(s)\right|^{p} \leq c_{11}+c_{12} \int_{0}^{s}\left|u^{n}(t)\right|^{p} d t
$$

because $F^{n} \tilde{\varphi} \geq 0$.

Now, by Gronwall's inequality we can conclude

$$
\left|u^{n}(s)\right| \leq C_{1}
$$

which together with (3.15) implies

$$
\int_{0}^{s}\left[u^{n}(t)\right]^{p} d t \leq C_{2}
$$

From (3.17), (3.18) and (H4) we deduce that

$$
\int_{0}^{s}\left\|u^{n}(t)\right\|^{p} d t \leq C_{3}
$$

and then

$$
\int_{0}^{s} F^{n}(t) \tilde{\varphi}\left(\Lambda_{E}^{-1} B^{*} u^{n}(t)\right) d t \leq C_{4}
$$

Finally, by using (3.19) and Lemma 1 for (3.12), we obtain

$$
\int_{0}^{T}\left(\phi\left(u^{n}(t)\right)+F^{n}(t) \tilde{\varphi}\left(\Lambda_{E}^{-1} B^{*} u^{n}(t)\right) d t \leq C_{5}\right.
$$

and then

$$
\int_{0}^{T}\left|\psi^{n}\left(t, u^{n}(t)\right)\right| d t \leq C_{6}
$$

A priori estimates II. - Multiplying (3.10) by $F^{n}(t)$ we obtain

$$
\begin{gathered}
F^{n}(t)\left(\frac{d u^{n}}{d t}(t), \frac{d u^{n}}{d t}(t)\right)+F^{n}(t)\left(\partial \psi^{n}\left(t, u^{n}(t)\right), \frac{d u^{n}}{d t}(t)\right)= \\
=F^{n}(t)\left(f(t), \frac{d u^{n}}{d t}(t)\right)
\end{gathered}
$$


On the other hand we have

$$
\begin{gathered}
\left(\partial \psi^{n}\left(t, u^{n}(t)\right), \frac{d u^{n}}{d t}(t)=\frac{d}{d t} \psi^{n}\left(t, u^{n}(t)\right)-\right. \\
-F^{\prime}(t) \varphi\left(\Lambda_{E^{-1}}^{-1} B^{*} u^{n}(t)\right)
\end{gathered}
$$

Integrating (3.23) between 0 and $T$ and using (3.24) we deduce

$$
\begin{aligned}
& \frac{1}{2} \int_{0}^{T} F^{n}(t)\left|\frac{d u^{n}}{d t}(t)\right|^{2} d t+F^{n}(T) \psi^{n}\left(T, u^{n}(T)\right) \\
& =F^{n}(0) \psi^{n}\left(0, u_{0 n}\right)+\int_{0}^{T} F^{\prime}(t) \psi^{n}\left(t, u^{n}(t)\right) d t \\
& +\int_{0}^{T} F^{n}(t)\left(f, \frac{d u^{n}}{d t}(t)\right) d t+\int_{0}^{T} F^{\prime}(t) F^{n}(t) \varphi\left(\Lambda_{E}^{-1} B^{*} u^{n}(t)\right) d t
\end{aligned}
$$

Now, using lemma 1, (H8) and the a priori estimates $I$ (note that $\left.F^{n}(0) \psi^{n}\left(0, u_{0_{n}}\right) \leq C_{7}\right)$, we obtain

$$
\begin{aligned}
& \frac{1}{2} \int_{0}^{T} F^{n}(t)\left|\frac{d u^{n}}{d t}(t)\right|^{2} d t+F^{n}(T) \phi\left(u^{n}(T)\right)+F^{n}(T)^{2} \tilde{\varphi}\left(\Lambda_{E}^{-1} B^{*} u^{n}(T)\right) \\
& \quad \leq d_{1}-F^{n}(T)^{2}\left(y, u^{n}(T)-v\right)+d_{2} \int_{0}^{F} F^{n}(t)\left|\frac{d u^{n}}{d t}(t)\right|^{2} d t
\end{aligned}
$$

where $d_{1}$ and $d_{2}$ denote positive constants. By using (H4), (H5) and Young's inequality we get

$$
\begin{aligned}
& d_{3} \int_{0}^{T} F^{n}(t)\left|\frac{d u^{n}}{d t}(t)\right|^{2} d t+F^{n}(T) \alpha\left[u^{n}(T)\right]^{p} \\
& \leq d_{4}+d_{5}\left|u^{n}(T)\right|^{p}+d_{6} \epsilon\left[u^{n}(T)\right]^{p}
\end{aligned}
$$

Finally choosing a suitable $\epsilon$, we obtain

$$
\int_{0}^{T} F^{n}(t)\left|\frac{d u^{n}}{d t}(t)\right|^{2} d t \leq C_{8}
$$

Passing to the limit $(n \rightarrow \infty) .-B y(3.17),(3.19)$ and (3.28), the sequence $\left\{u^{n}\right\}$ has a subsequence $\left\{u^{k}\right\}$ such that

$$
\left\{u^{k}\right\} \rightarrow u \quad \text { in } L^{p}(0, T ; V) \quad \text { weakly }
$$




\section{A. Bermudez and C. Rodriguez}

$$
\begin{gathered}
\left\{u^{k}\right\} \rightarrow u \quad \text { in } L^{\infty}(0, T ; H) \quad \text { weakly-star } \\
\left\{\sqrt{F} \frac{d u^{k}}{d t}\right\} \rightarrow \sqrt{F} \frac{d u}{d t} \text { in } L^{2}(0, T ; H) \quad \text { weakly }
\end{gathered}
$$

From (3.10). we get

$$
\begin{aligned}
F^{k}(t)^{2}\left(f, z(t)-u^{k}(t)\right) & \leq F^{k}(t)^{2}\left(\frac{d u^{k}}{d t}(t), z(t)-u^{k}(t)\right)+F^{k}(t)^{2} \phi(z(t)) \\
& -F^{k}(t)^{2} \phi\left(u^{k}(t)\right)+F^{k}(t)^{3} \varphi\left(\Lambda_{E}^{-1} B^{*} z(t)\right)- \\
& -F^{k}(t)^{3} \varphi\left(\Lambda_{E}^{-1} B^{*} u^{k}(t)\right), \forall z \in L^{2}(0, T ; V)
\end{aligned}
$$

Moreover, we have

a) $\quad \limsup \left(-\int_{0}^{T} F^{k}(t)^{2} \phi\left(u^{k}(t)\right) d t-\int_{0}^{T} F^{k}(t)^{3} \varphi\left(\Lambda_{E}^{-1} B^{*} u^{k}(t)\right) d t\right.$

$$
\leq-\int_{0}^{T} F(t)^{2} \phi(u(t)) d t-\int_{0}^{T} F(t)^{3} \varphi\left(\Lambda_{E}^{-1} B^{*} u(t)\right) d t
$$

by using the lower-semicontinuity of $\phi$ and $\varphi$.

b)

$$
\begin{gathered}
\limsup \int_{0}^{T} F^{k}(t)^{2}\left(\frac{d u^{k}}{d t}(t), z(t)-u^{k}(t)\right) d t= \\
=\int_{0}^{T} F(t)^{2}\left(\frac{d u}{d t}(t), z(t)-u(t)\right) d t
\end{gathered}
$$

To prove (3.34) we consider the equality

$$
F^{k}(t)^{2}\left(\frac{d u^{k}}{d t}(t), u^{k}(t)\right)=\left(\sqrt{F^{k}(t)} \frac{d u^{k}}{d t}(t), F^{k}(t)^{3 / 2} u^{k}(t)\right)
$$

Moreover, we have the following facts :

i) $\left(F^{k}(t)^{3 / 2}\right)^{\prime} u^{k}(t)$ is bounded in $L^{2}(0, T ; H)$ since (3.17) and (H8) hold. From (3.28) and (H8) we derive that $F^{k}(t)^{3 / 2} d u^{k} / d t$ is bounded in $L^{2}(0, T ; H)$ and then we have

$$
\frac{d}{d t}\left(F^{k}(t)^{3 / 2} u^{k}(t)\right) \text { is bounded in } L^{2}(0, T ; H)
$$

ii) From (3.17) and (H8) we can conclude that

$$
F^{k}(t)^{3 / 2} u^{k}(t) \text { is bounded in } L^{2}(0, T ; H)
$$


Now, taking into account (3.36) and (3.37) and using a theorem on compact imbeddings (see for instance [11] p. 57) we have

$$
F^{k}(t)^{3 / 2} u^{k}(t) \rightarrow F^{3 / 2} u(t) \text { in } L^{2}(0, T ; H) \text { strongly }
$$

From (3.35), (3.31) and (3.38) we can conclude (3.34).

Integrating (3.32) between 0 and $T$ and taking the limsup, $k \rightarrow \infty$, we can pass to the limit in (3.32). Next, by classical arguments we obtain the corresponding pointwise inequality a.e. on $(0, T)$. Finally we can eliminate the term $F(t)^{2}$, because $F(t) \neq 0$ a.e and we can conclude that $u$ is a solution of (3.1).

Uniqueness. - It follows from the monotonicity of $\phi^{\prime}$ and $\partial \varphi$ in a classical way.

\section{Numerical solution}

In order to solve the inequality (3.1) we shall make two discretizations, one the variable $t$ and another one in the spaces $V, H, \mathbf{R}$.

Discretizations in $t$. - Let $M$ be a natural number and $k=T / M$. We denote by $u^{n}$ the "approximation" of $u$ at time $t_{n}=n k$.

Introduce

$$
f^{n+1}=\int_{n k}^{(n+1) k} f(t) d t \text { and } F^{n+1}=F\left(t_{n+1}\right)
$$

We obtain $u^{n}$ as the solution of the following problem $\left(P^{k}\right):$ Find $\left\{u^{n} \in\right.$ $V: 0 \leq n \leq M-1\}$ such that

$$
\left\{\begin{array}{l}
\left(\frac{u^{n+1}-u^{n}}{k}, z-u^{n+1}\right)+\phi(z)-\phi\left(u^{n+1}\right)+F^{n+1} \varphi\left(\Lambda_{E}^{-1} B^{*} z\right)- \\
\quad-F^{n+1} \varphi\left(\Lambda_{E}^{-1} B^{*} u^{n+1}\right) \geq\left(f^{n+1}, z-u^{n+1}\right) \quad \forall z \in V \\
u^{0}=u_{0}
\end{array}\right.
$$

It is known that this problem has a unique solution.

Discretization in space. - We solve the problem $\left(P^{k}\right)$ for the particular case of the Marangoni effect (see Remark 1).

For the discretization $V$ and $H$, we use picewise linear Lagrange Finite elements, more precisely we replace $V$ and $H$ by the following

$$
\begin{aligned}
& V_{h}=\left\{v \in C^{0}(\bar{\Omega}):\left.v\right|_{K} \in P_{1} \forall K \in \tau_{h}, v(L)=0\right\} \\
& H_{h}=\left\{v:\left.v\right|_{K} \in P_{0} \quad \forall K \in \tau_{h}\right\}
\end{aligned}
$$


where $\tau_{h}$ is a mesh of $\Omega$, with $h=L /(N+1), N \in \mathbf{N}$.

We consider the following discrete problem $\left(P_{h}^{k}\right)$ Find $\left\{u_{h}^{n} \in V_{h}: 0 \leq\right.$ $n \leq M-1\}$ such that

$$
\left\{\begin{array}{l}
\left(\frac{u_{h}^{n+1}-u_{h}^{n}}{k}, z_{h}-u_{h}^{n+1}\right)+\phi\left(z_{h}\right)-\phi\left(u_{h}^{n+1}\right)+F^{n+1} \varphi\left(\Lambda_{E}^{-1} B^{*} z_{h}\right)- \\
\quad-F^{n+1} \varphi\left(\Lambda_{E}^{-1} B^{*} u_{h}^{n+1}\right) \geq\left(f^{n+1}, z_{h}-u_{h}^{n+1}\right) \quad \forall z_{h} \in V_{h} \\
u^{0}=u_{0 h}
\end{array}\right.
$$

For problem $\left(P_{n}^{k}\right)$, we also have existence and uniqueness of solution.

Let $\gamma$ be the operator in $H_{h}$ defined by

$$
\gamma\left(z_{h}\right)=\left|z_{h}\right|^{p-2} z_{h}
$$

Note that $\gamma$ and $G$ given by (2.3) are maximal monotone operators in $\mathbf{R}^{2}$ and $\mathbf{R}$ respectively.

From (4.1) we deduce the existence of $q_{h}^{n+1} \in \mathbf{R}$ and $p_{h}^{n+1} \in H_{h}$ such that

$$
\begin{gathered}
p_{h}^{n+1} \in \gamma\left(\frac{d u_{h}^{n+1}}{d x}\right)-w_{1} \frac{d u_{h}^{n+1}}{d x} \\
q_{h}^{n+1} \in G\left(u_{h}^{n+1}(0)\right)-w_{2} u_{h}^{n+1}(0) \\
\int_{0}^{L} \frac{u_{h}^{n+1}}{k} v_{h} d x+w_{1} \int_{0}^{L} \frac{d u_{h}^{n+1}}{d x} \frac{d v_{h}}{d x} d x+w_{2} u_{h}^{n+1}(0) F^{n+1} v_{h}(0)= \\
=-\int_{0}^{L} p_{h}^{n+1} \frac{d v_{h}}{d x} d x-q_{h}^{n+1} F^{n+1} v_{h}(0)+\int_{0}^{L} \frac{u_{h}^{n}}{k} v_{h} d x+\int_{0}^{L} f^{n+1} v_{h} d x \\
\forall v_{h} \in \tau_{h}
\end{gathered}
$$

where $w_{1}$ and $w_{2}$ are arbitrarily given positive constants.

On the other hand, from (4.3) and (4.4) (see [4], [5]) we deduce

$$
\begin{aligned}
& p_{h}^{n+1}=\rho_{1}\left(\gamma_{\lambda_{1}}^{w_{1}}\left(\frac{d u_{h}^{n+1}}{d x}+\lambda_{1} p_{h}^{n+1}\right)\right)+\left(1-\rho_{1}\right) p_{h}^{n+1} \\
& q_{h}^{n+1}=\rho_{2}\left(G_{\lambda_{2}}^{w_{2}}\left(u_{h}^{n+1}(0)+\lambda_{2} q_{h}^{n+1}\right)\right)+\left(1-\rho_{2}\right) q_{h}^{n+1}
\end{aligned}
$$

where $\gamma_{\lambda_{1}}^{w_{1}}$ and $G_{\lambda_{2}}^{w_{2}}$ denote the Yosida approximations of $\gamma-w_{1} I$ and $G-w_{2} I$ respectively. They are given by the following expressions

$$
\begin{gathered}
\gamma_{\lambda_{1}}^{w_{1}}(z)=\frac{1}{\lambda_{1}}\left(z-J_{\frac{\lambda_{1}}{1-\lambda_{1} w_{1}}}^{\gamma}\left(\frac{z}{1-\lambda_{1} w_{1}}\right)\right) \\
-216-
\end{gathered}
$$




$$
G_{\lambda_{2}}^{w_{2}}(x)=\frac{1}{\lambda_{2}}\left(x-J_{\frac{\lambda_{2}}{1-\lambda_{2} w_{2}}}^{G}\left(\frac{x}{1-\lambda_{2} w_{2}}\right)\right)
$$

where $J_{\frac{\lambda_{1}}{1-\lambda_{1} \omega_{1}}}^{\gamma}$ and $J_{\frac{\lambda_{2}}{1-\lambda_{2} \omega_{2}}}$ are the resolvents of the operators $\gamma$ and $G$ respectively.

For $\gamma$ and $G$ given by (4.2) and (2.3), we have

$$
J_{\mu}^{\gamma}(z)=\alpha \frac{z}{|z|}
$$

where $\alpha$ is a solution of the equation $\mu \alpha^{p-1}+\alpha-|z|=0$ (we can compute $\alpha$ by Newton's method), and

$$
J_{\mu}^{G}(x)=\frac{x-\sqrt{x^{2}+4 \mu}}{2}
$$

The formulation (4.5), (4.6) and (4.7) leads to the following algorithm $q_{h 0}^{n+1} \in \mathbf{R}$ and $p_{h 0}^{n+1} \in H_{h}$ arbitrarily chosen,

$$
\begin{aligned}
& \int_{0}^{L} \frac{u_{h r}^{n+1}}{k} v_{h} d x+w_{1} \int_{0}^{L} \frac{d u_{h r}^{n+1}}{d x} \frac{d v_{h}}{d x} d x+w_{2} u_{h}^{n+1}(0) F^{(N+1)} v_{h}(0)= \\
& =-\int_{0}^{L} p_{h r}^{n+1} \frac{d v_{h}}{d x} d x-q_{h r}^{n+1} F^{N+1} v_{h}(0)+\int_{0}^{L} \frac{u_{h}^{n}}{k} v_{h} d x+\int_{0}^{L} f^{n+1} v_{h} d x
\end{aligned}
$$

$$
\begin{aligned}
& p_{h r}^{n+1}=\rho_{1} \gamma_{\lambda_{1}}^{w_{1}}\left(\frac{d u_{h r}^{n+1}}{d x}+\lambda_{1} p_{h r}^{n+1}\right)+\left(1-\rho_{1}\right) p_{h r}^{n+1} \\
& q_{h r}^{n+1}=\rho_{2} G_{\lambda_{2}}^{w_{2}}\left(u_{h r}^{n+1}(0)+\lambda_{2} q_{h r}^{n+1}\right)+\left(1-\rho_{2}\right) q_{h r}^{n+1}
\end{aligned}
$$

Note that (4.12) is a linear problem with a constant matrix which is computed and factorized only once.

The convergence of (4.13) and (4.14) is proved in Bermudez [4].

The following figures show the numerical results optained for the test examples

\section{Test 1}

$$
\begin{aligned}
& \nu=1 \\
& T=1.5 \\
& L=2 \\
& p=2
\end{aligned}
$$




\section{A. Bermudez and C. Rodriguez}

$$
\begin{aligned}
& f(x, t)=-e^{-x} 2(t-1) e^{-e(t-1)^{2}}-e^{-x}\left(e^{-(t-1)^{2}}-e^{-1}\right) \\
& F(t)=\left(e^{-(t-1)^{2}}-e^{-1}\right)^{2} \\
& u(x, 0)=0 \\
& u(L, t)=e^{-L}\left(e^{-(t-1)^{2}}-e^{-1}\right) \\
& \text { exact solution : } u(x, t)=e^{-x}\left(e^{-(t-1)^{2}}-e^{-1}\right)
\end{aligned}
$$

\section{Test 2}

$$
\begin{aligned}
& \nu=1 \\
& T=1.5 \\
& L=2 \\
& f(x, t)=e^{-x} e^{t}-(p-1)\left(e^{-x}\left(e^{t}-1\right)\right)^{p-1} \\
& F(t)=\left(e^{t}-1\right)^{p} \\
& u(x, 0)=0 \\
& u(L, t)=e^{-L}\left(e^{t}-1\right) \\
& \text { exact solution : } u(x, t)=e^{-x}\left(e^{t}-1\right)
\end{aligned}
$$




\section{A nonlinear evolution equation modelling}
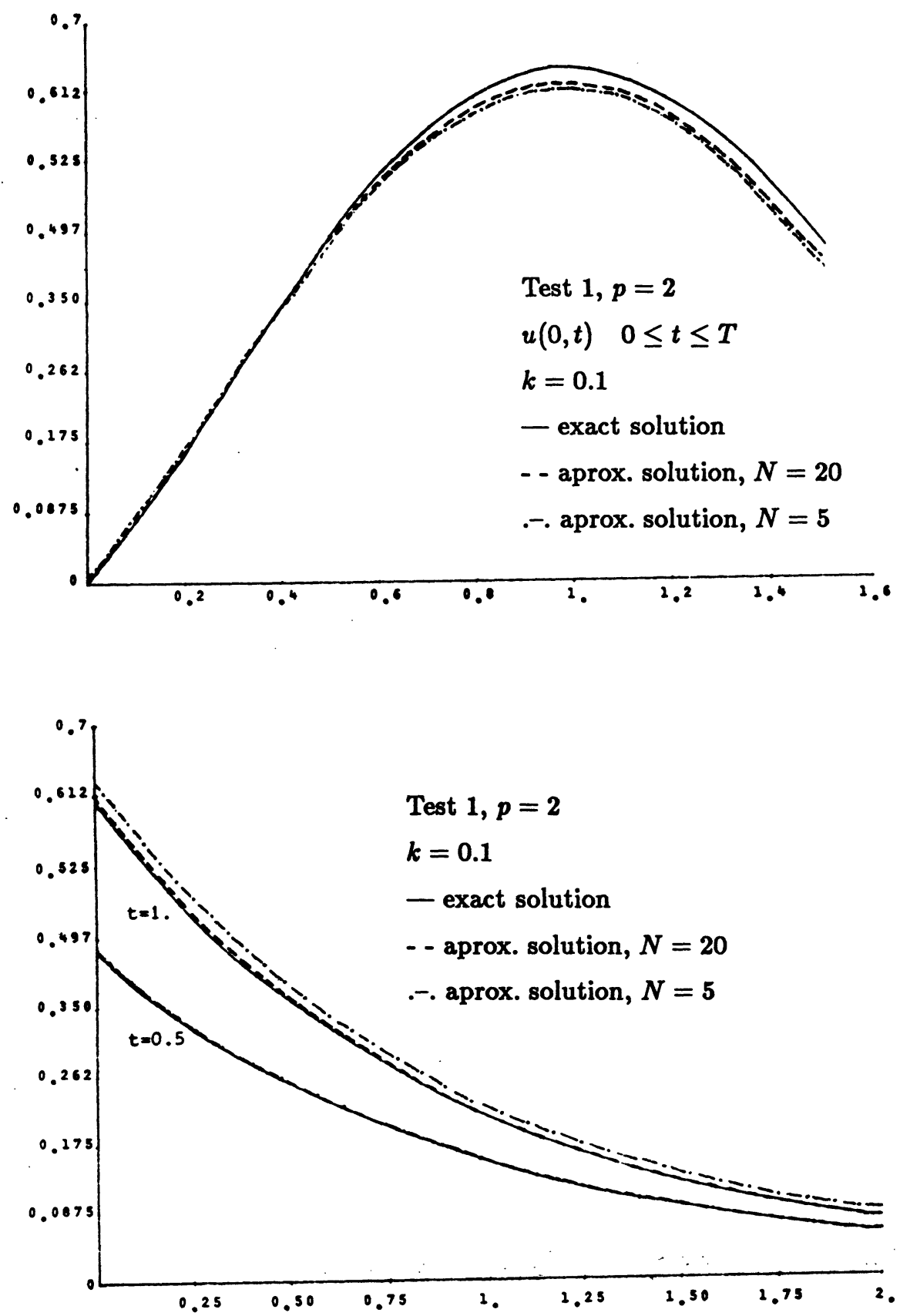
A. Bermudez and C. Rodriguez
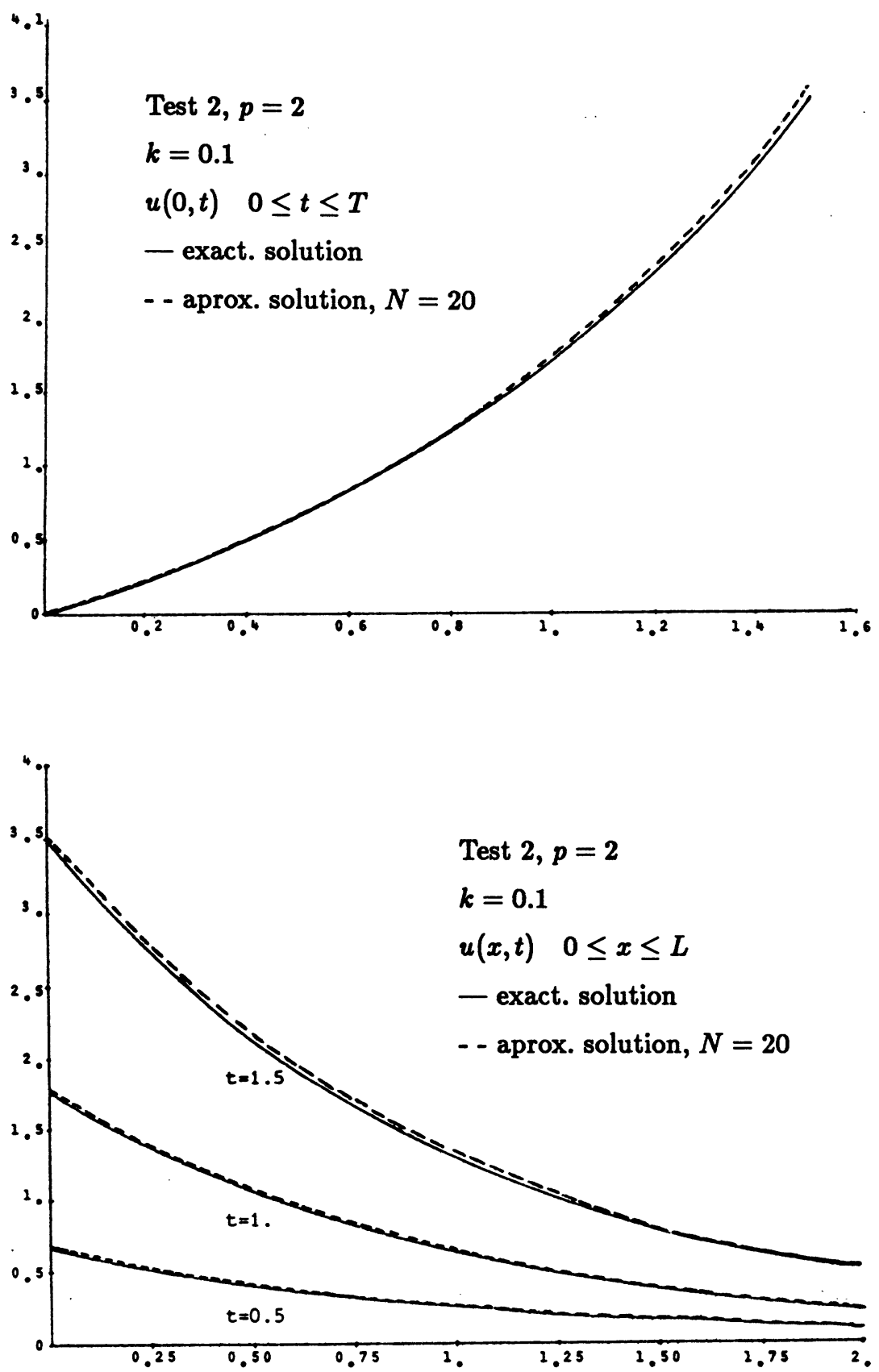
A nonlinear evolution equation modelling
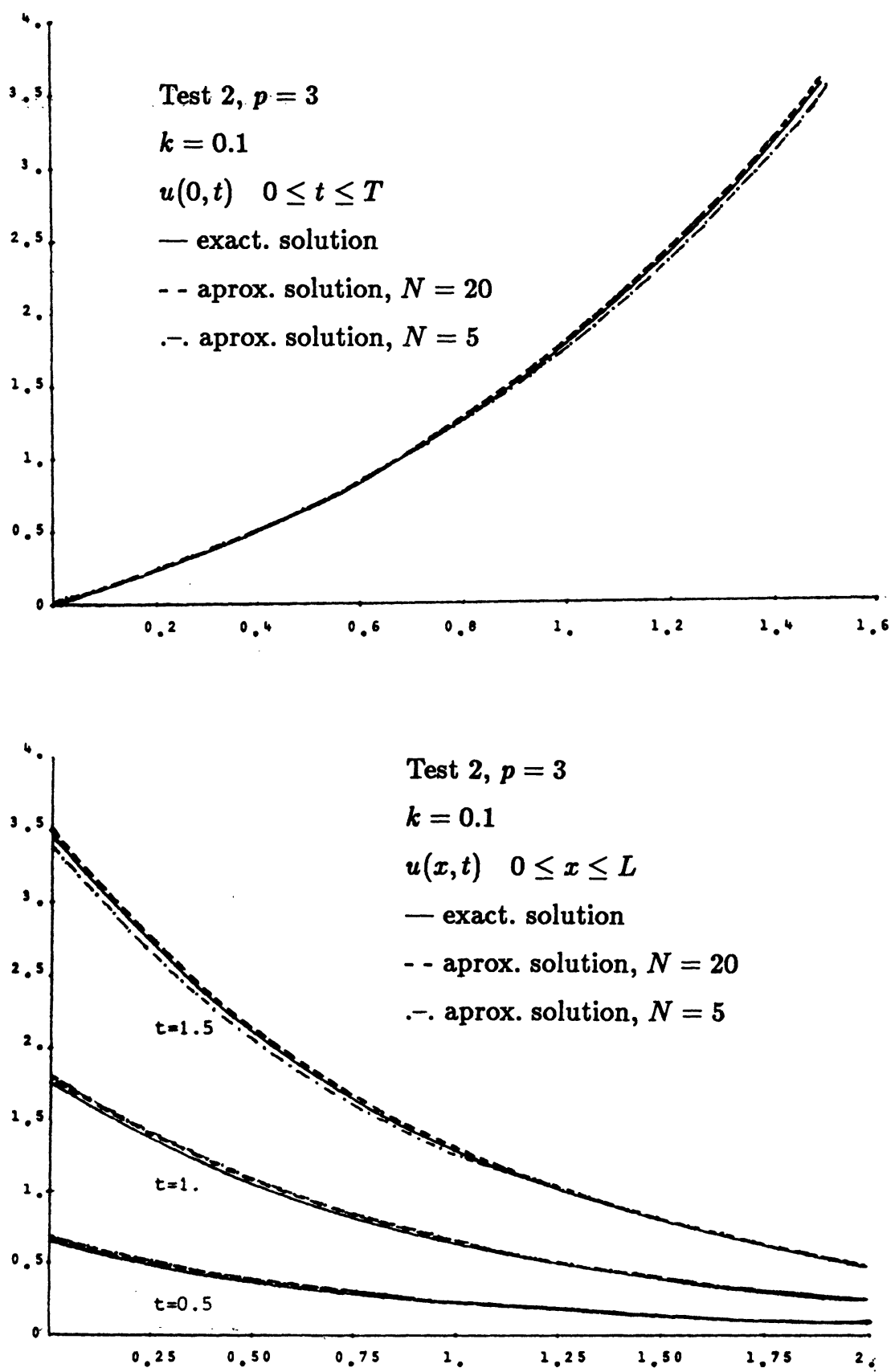
A. Bermudez and C. Rodriguez
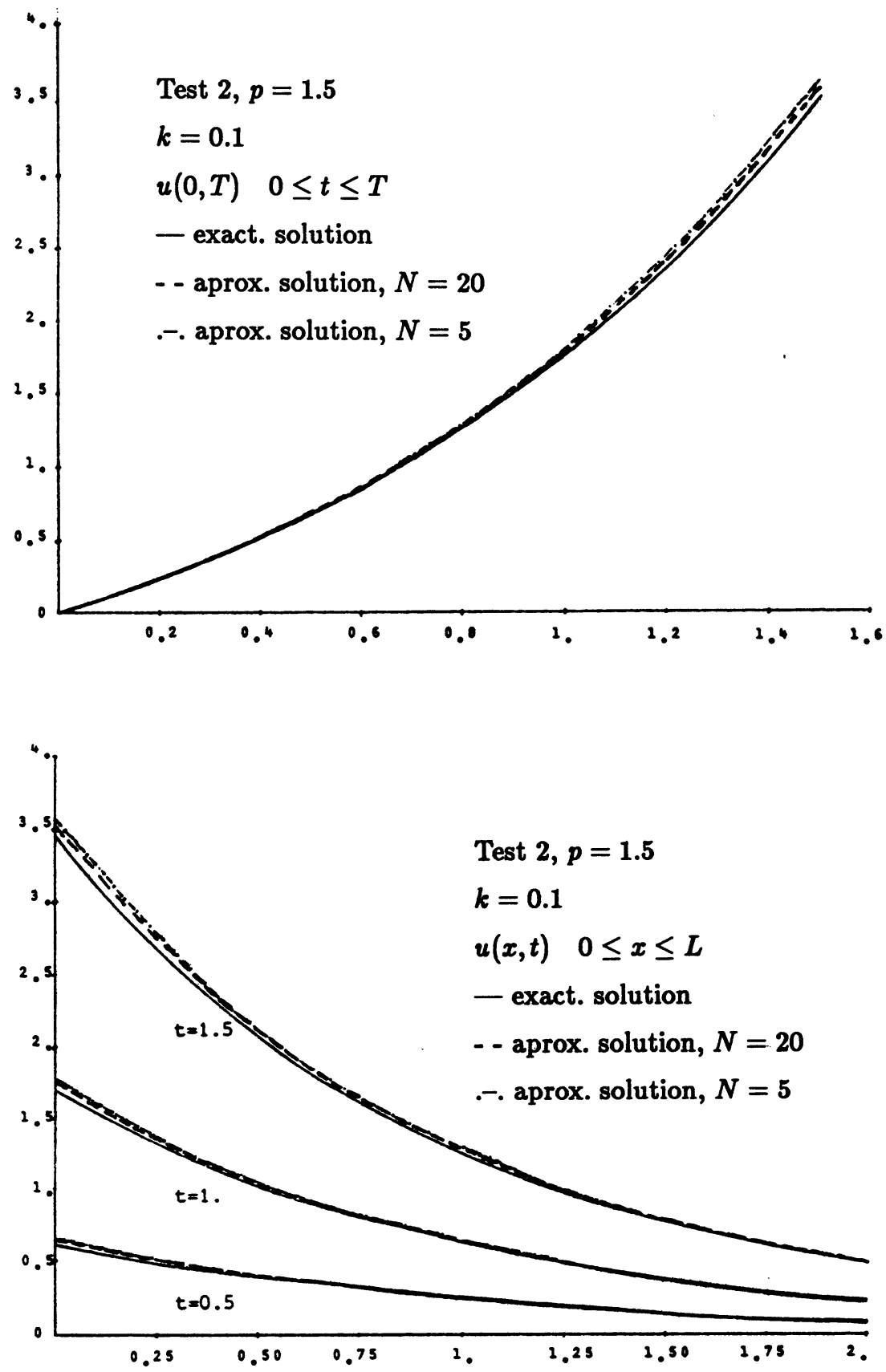
Acknowledgement. - We wich to thank Prof. A. Damlamian for their valuables suggestions and comments on this paper.

This work was partially supported by the C.A.Y.C.I.T. (Project $\mathrm{n}^{\circ} 1800-$ 82).

\section{Réferences}

[1] Attouch (H.) and Damlamian (A.). - Problèmes d'évolution dans les Hilberts et applications, J. Math. Pures et Appl., t. 54, 1975, p. 53-74.

[2] Attouch (H.) and Damlamian (A.). - Strong solution for parabolic variational inequalities, Nonlinear Anal., Theory, Methods and Applications, t. II, 3, 1978, p. 329-353.

[3] BARbu (V.) and Precupanu (T.).- Convexity and optimization in Banach spaces. - Academiei Bucuresti (Romania), 1978.

[4] Bermudez (A.). - Un método numérico para la resolucion de ecuaciones con varios términos no lineales. Applicacion a un problema de flujo de gas en un conducto, Real Academia de Ciencias Exactas, Fisicas y Naturales de Madrid, Vol. LXXVII, 4, 1984 .

[5] BERMUdez (A.) and MORENO (C.).-Duality methods for solving variational inequalities, Comput. and Math. Appl., t. 7, 1981, p. 43-58.

[6] Bermudez (A.), Durany (J.) and Saguez (C.). - An existence theorem for an implicit nonlinear evolution equation. - Collectanea Mathematica, Vol. XXXV, 1 Barcelona, (Espana), 1984.

[7] BREzIs (H.). - Operateurs maximaux monotones et semi-groupes de contractions dans les espaces de Hilbert. - North-Holland, London, 1973.

[8] CRANdall (M.) and PAzY (A.). - Nonlinear evolution equations in Banach spaces, Israel J. Math., t. 11, 1972, p. 57-94.

[9] KATO (T.). - Nonlinear semi-groups and evolution equations, J. Math. Soc. Japan, t. 19,1967, p. $508-520$.

[10] KenMochi (N.). - The semi-discretisation method and nonlinear time-dependent parabolic variational inequalities, Proc. Japan Acad., t. 50, 1975, p. 714-717.

[11] Lions (J.L.). - Quelques méthodes de résolution des problèmes aux limites non linéaires. - Dunod, Paris, 1969.

[12] Peralba (J.C.). - Un problème d'évolution relatif à un opérateur sous-différentiel dépendant du temps, C.R. Acad. Sci., Paris, t. 275, 1872, p. 93-96.

[13] Ruckenstein (E.), SMrgelschi (O.) and Suciu (D.G.). - A steady dissolving drop method for studying the pure Marangoni effect, Chemical Engineering Science, t. 25, 1970, p. 1249-1254.

[14] WatANA BE (J.). - On certain nonlinear evolution equations, J. Math. Soc. Japan, t. 25, 1973, p. 446-463. 